\title{
Impact of dilute acid pretreatment conditions on p-coumarate removal in diverse maize lines
}

\author{
Authors: Brian K. Saulnier, Thanaphong \\ Phongpreecha, Sandip K. Singh, David B. Hodge
}

(C) 2020 This manuscript version is made available under the CC-BY-NC-ND 4.0 license https:// creativecommons.org/licenses/by-nc-nd/4.0/

Saulnier, Brian K., Thanaphong Phongpreecha, Sandip K. Singh, and David B. Hodge. "Impact of Dilute Acid Pretreatment Conditions on p-Coumarate Removal in Diverse Maize Lines." Bioresource Technology 314 (October 2020): 123750. doi:10.1016/j.biortech.2020.123750 


\title{
Impact of Dilute Acid Pretreatment Conditions on $p$-Coumarate Removal in Diverse Maize Lines
}

Brian K. Saulnier ${ }^{1}$, Thanaphong Phongpreecha ${ }^{2}$, Sandip K. Singh ${ }^{1}$, David B. Hodge ${ }^{1,3^{*}}$

${ }^{1}$ Department of Chemical \& Biological Engineering, Montana State University, Bozeman, Montana 59717, United States

${ }^{2}$ School of Medicine, Stanford University, Stanford, California 94305, United States

${ }^{3}$ Division of Sustainable Process Engineering, Luleå University of Technology, Luleå 97187, Sweden

* corresponding author

Email: $\underline{\text { david.hodge3@montana.edu }}$

\begin{abstract}
Prior work has identified that lignins recovered from dilute acid-pretreated corn stover exhibit superior performance in phenol-formaldehyde resins used in wood adhesive applications when compared to diverse process-modified lignins derived from other sources. This improved performance is hypothesized to be due to the higher content of unsubstituted phenolic groups specifically $p$-coumarate lignin esters. In this work, a diverse set of corn stover samples are employed that exhibit diversity in $p$-coumarate content and total lignin content to explore the relationship between dilute acid pretreatment conditions, $p$-coumarate ester hydrolysis, xylan solubilization, and the resulting glucose enzymatic hydrolysis yields. The goal of this study is to identify pretreatment conditions that preserve a significant fraction of the $p$-coumarate esters while simultaneously achieving high enzymatic hydrolysis yields. Kinetic parameters for pcoumarate ester hydrolysis were quantified and pretreatment-biomass combinations were identified that result in glucose hydrolysis yields of more than $90 \%$ while retaining nearly $50 \mathrm{mg}$ $p$-coumarate/g lignin.
\end{abstract}

Keywords: Corn stover; dilute acid pretreatment; $p$-coumarate; lignin 


\section{Introduction}

Technologies for the production of cellulosic biofuels from herbaceous feedstocks which utilize a pretreatment and enzymatic hydrolysis have begun to be commercialized in the last few years (Warner et al., 2017). However, there are still technological and economic challenges that limit widespread adoption of these biomass conversion routes. One approach to improving the process economics of the lignocellulose to biofuels processes is through diversification of the product portfolio to include renewable, bio-based chemicals and materials (Shanks and Keeling, 2017). This approach is widely recognized as an opportunity both to improve the economics of these processes and to buffer against market fluctuations. In particular, there is an opportunity for technologies that can be integrated with conversion processes which employ a biomass pretreatment/fractionation strategy, and allow for the utilization of all or a fraction of the process-modified lignins for purposes other than as a solid fuel for process heat and power.

Lignin represents one of the few renewable sources of aromatic chemicals, and lignin valorization has been a subject of increasing recent research to enable the use of lignin as a feedstock for bio-based products (Ragauskas et al., 2014). Lignins may make up more than $1525 \%$ of the mass of the feedstock entering a biorefinery; the extraction of additional value from these biorefinery-derived lignins provides an opportunity to improve the economic competitiveness of these facilities. Commercialization of co-products from wood lignins derived from Kraft pulping has long proved challenging. However a lignin's origin (i.e., hardwood, softwood, or grass) and processing history (i.e., alkaline pulping, organosolv pulping, sulfite pulping, dilute acid pretreatment, etc.) have an enormous impact on the structure, properties, and suitability of the lignin for a particular application. Dilute acid pretreatment has been studied for many decades at both the pilot (Schell et al., 2003) and demonstration scale (Carlson, 2020). However, the use of the water-insoluble lignin remaining after enzymatic hydrolysis following dilute acid pretreatment has been limited in practice to use as a boiler fuel.

As motivation for this work, it was recently demonstrated that a purified subset of dilute acid-pretreated corn stover lignin derived from the enzymatic hydrolysis residue (i.e., the "lignin cake”) from a demonstration-scale biorefinery could be used as a $100 \%$ phenol replacement in PF adhesives for plywood manufacturing with performance comparable to a commercial phenol resorcinol formaldehyde adhesive (Kalami et al., 2017). This is notable in that these results could not be achieved using other process lignins including softwood and hardwood lignins derived 
from Kraft pulping, lignins derived from the organosolv pulping of softwood, hardwood, corn stover, and wheat straw lignin derived from soda pulping (Kalami et al., 2018). It has been hypothesized that the high quantified content of unsubstituted phenolic groups in the corn stover lignins, presumably as $p$-coumarate ( $p \mathrm{CA}$ ), are responsible for this lignin's high level of incorporation into the PF resin polymer matrix.

A significant number of promising bioenergy feedstocks are graminaceous monocots or grasses including agricultural residues such as corn stover, wheat straw, rice straw, and sugar cane bagasse. One distinguishing feature of the graminaceous monocot lignins is the considerable incorporation of the $p$-hydroxycinnamic acids including ferulate and $p \mathrm{CA}$ (Carpita, 1996; Hatfield et al., 1999). It is known that $p \mathrm{CA}$ is acylated to the lignin polymer at the $\gamma$ carbon of the side chain region primarily in syringyl moieties and to a lesser degree on arabinosyl side chains of arabinoxylan (Hatfield et al., 2008). The $p$ CA content of grasses is known to exhibit substantial diversity with respect to genotype, anatomical fraction, and maturity (Li et al., 2015; Li et al., 2017) with the content in some fractions in maize reaching more than $150 \mathrm{mg} p \mathrm{CA} / \mathrm{g}$ Klason lignin (Hatfield et al., 2008) or $\sim 13 \%$ of the total aromatic content of the biomass. The prior processing history of the biomass (i.e., pretreatment) as well as any lignin recovery and purification steps will also impact the $p \mathrm{CA}$ content of the lignin as the ester bond is labile to acid- or base-catalyzed hydrolysis. Therefore, if the hypothesis that $p \mathrm{CA}$ content is the most important contributor to lignin suitability in PF resins is correct, then the feedstock genotype, together with growth conditions, harvest time, and lignin processing history are all of critical importance for lignin suitability as a raw material for these applications.

In this study we seek to further understand the fate of $p \mathrm{CA}$ in corn stover lignins during dilute acid pretreatment with the goal of identifying how both the feedstock and the processing conditions could be optimally coupled to extract the most value from the lignin co-product. Specifically, we investigate the impact of plant genotype in diverse maize lines of differing initial lignin, $p \mathrm{CA}$ contents, and dilute acid pretreatment conditions (pretreatment time and initial acid loading) on the response of the biomass to pretreatment (composition and enzymatic hydrolysis yields) with the goal of identifying the balance between high hydrolysis yields and low $p$ CA solubilization (i.e., higher quality lignin for co-products). Finally, we performed the acid-catalyzed hydrolysis of a model $p C A$ ester under conditions comparable to dilute acid pretreatment and estimated kinetic parameters for ester hydrolysis. 


\section{Materials and Methods}

\subsection{Biomass Feedstock}

A panel of inbred maize (Zea mays L.) lines were used as a biomass source and represent a subset of a wider diversity panel grown in Arlington, Wisconsin as reported in our previous work (Li et al., 2015). These include lines B7, F431, NC368, B14A, NK807, and 5220 and were selected based on extremes in the contents of lignin and $p \mathrm{CA}$.

\section{$\underline{2.2} \underline{\text { Pretreatment Conditions }}$}

Pretreatments were conducted at 10\% solid loadings, (g corn stover/g liquid solution) in dilute $\mathrm{H}_{2} \mathrm{SO}_{4}$ solution using $75-\mathrm{mL}$ round bottom ChemGlass pressure vessels immersed in a temperature-controlled silicone oil bath heated by a hot plate. Dilute acid solutions varied in initial $\mathrm{pH}$ between 2.36 and 3.66 and treatment times between 45 and 360 minutes at $180^{\circ} \mathrm{C}$ with stirring at $260 \mathrm{rpm}$. Treated biomass was separated from reaction liquor via vacuum filtration, washed with $500 \mathrm{~mL}$ of water, and air dried for 4 days at room temperature. Biomass yields after pretreatment were determined gravimetrically.

\section{$\underline{2.3}$ Composition Analysis}

Klason lignin and sugar content of biomass was determined using the NREL laboratory analytical procedure LAP, TP-510-42618 (Sluiter et al., 2008). Sugars were quantified by HPLC using a Bio-Rad Aminex HPX-87H column with a mobile phase of $5 \mathrm{mM} \mathrm{H}_{2} \mathrm{SO}_{4}$ at a flow rate of $0.6 \mathrm{~mL} / \mathrm{min}$ and $\mathrm{a} 65^{\circ} \mathrm{C}$ column temperature with quantification using RID detection. Remaining moisture content of biomass was determined gravimetrically after drying the biomass for 4 hours at $105^{\circ} \mathrm{C}$. Ash content was determined gravimetrically after heating the biomass at $620^{\circ} \mathrm{C}$ for 4 hours according to the NREL LAP TP-510-42622 (Sluiter et al., 2008).

\section{$\underline{2.4}$ Esterified p-Coumarate Content}

Alkali-hydrolyzable $p$-coumarate content was determined via alkaline saponification. Biomass (250 mg) was placed in 40-mL Teflon-lined stainless-steel synthesis reactors (Shenzen Langce Ltd., Huizhou, China) with $12.5 \mathrm{~mL}$ of $3 \mathrm{M} \mathrm{NaOH}$. These reactors were heated to $180^{\circ} \mathrm{C}$ for 2 hours with a stir rate of $280 \mathrm{rpm}$. After cooling, alkaline liquor was adjusted to below pH 2 
using $150 \mu \mathrm{L} \mathrm{H}_{2} \mathrm{SO}_{4}$ and centrifuged at 13,400 rpm (10,400 x g) for 10 minutes to remove the precipitate from the supernatant. Cinnamic acids in the supernatant were quantified using an Agilent Poroshell $120 \mathrm{C} 18$ column using a gradient of two solvents, $1 \%$ acetic acid in water (solvent A), and 1\% acetic acid in 50/50\% v/v methanol and water (solvent B). A 32-minute gradient was used reducing from $100 \%$ solvent $\mathrm{A}$ to $30 \%$ solvent $\mathrm{A}$ and $70 \%$ solvent $\mathrm{B}$ over 2 minute increments with at a flow rate of $0.4 \mathrm{~mL} / \mathrm{min}$ and a column temperature of $40^{\circ} \mathrm{C}$. Acids were quantified using a DAD detector at $280 \mathrm{~nm}$.

\section{$\underline{2.5} \underline{\text { Enzymatic Hydrolysis }}$}

Enzymatic hydrolysis was conducted using CTec2 (Novozymes A/S, Bagsværd, Denmark) at an enzyme loading of $15 \mathrm{mg}$ protein/g glucan with a $10 \%$ solid biomass loading (g corn stover/g liquid). The hydrolysis was conducted in a $0.05 \mathrm{M}$ citrate buffer along with a 0.04 $\mathrm{mg} / \mathrm{mL}$ tetracycline solution to inhibit bacterial growth. Samples were placed in $15-\mathrm{mL}$ centrifuge tubes and subjected to orbital shaking in an incubator at $50^{\circ} \mathrm{C}$ for 24 hours. Sugar yields were calculated from the liquid phase glucose which was quantified using an Aminex HPX-87H column (Bio-Rad, Hercules, CA) with detection by RID. Glucose hydrolysis yields are calculated as the glucose released (as mass glucan) per mass glucan in the pretreated biomass based on composition analysis.

\subsection{Methyl trans-p-Coumarate Hydrolysis}

A solution of $0.8 \mathrm{mg} / \mathrm{mL}$ methyl trans-p-coumarate (TCI America, Portland, OR) was suspended in a solution of aqueous dilute sulfuric acid of $\mathrm{pH} 2.86$ and $\mathrm{pH} 3.36$ within $40-\mathrm{mL}$ Teflon-lined stainless-steel synthesis reactors. These reactors were heated to $180^{\circ} \mathrm{C}$ for times varying between 3 and 17 hours with a stir rate of $280 \mathrm{rpm}$ in a silicone oil bath. Decrease in reactant in the resulting reaction mixture was analyzed via HPLC using an Agilent Poroshell 120 C18 column. A 90 minute gradient from $100 \%$ solvent A to $70 \%$ solvent B and $30 \%$ solvent A with 6-minute increments was used with a flow rate of $0.4 \mathrm{~mL} / \mathrm{min}$ and a column temperature of $40^{\circ} \mathrm{C}$. Methyl trans $p$-coumarate was quantified using a DAD detector at $280 \mathrm{~nm}$. 


\section{Results and Discussion}

\section{$\underline{3.1}$ Biomass and Pretreatment}

The content of $p \mathrm{CA}$ within lignins has been proposed to be a key feature impacting the utility of dilute acid-pretreated corn stover lignins in phenol-formaldehyde resin applications (Kalami et al., 2018). Critically, $p$ CA should be substantially more reactive than other monomers within the lignin backbone as it has two ortho sites available to react with formaldehyde relative to the three positions available in phenol (two ortho and one para). As such, the impact of pretreatment conditions on the fate of $p \mathrm{CA}$ is an important, and yet largely unexplored topic for both understanding the mass flows within a biorefinery as well as the potential for increasing coproduct value. If the $p \mathrm{CA}$ content is important for lignin value in co-products applications, then the choice of conditions for pretreatments can be considered as a balance between (1) economic constraints, (2) conditions that optimize yields, and (3) conditions that preserve the $p \mathrm{CA}$ content in the lignin.

In addition to being impacted by processing, both Klason lignin content and $p \mathrm{CA}$ content are known to contribute to cell wall recalcitrance in corn stover. As an example, prior work with an expanded maize diversity panel demonstrated that both the initial $p \mathrm{CA}$ content and the initial Klason lignin content exhibited a statistically significant $(p<0.05)$ inverse correlation to glucose hydrolysis yields in untreated biomass subjected to enzymatic hydrolysis only (Ong et al., 2014).

In this study, one goal is to understand (1) how lignin content and $p$ CA content impact the response of the biomass to deconstruction using dilute acid pretreatment and enzymatic hydrolysis and (2) how lignin-associated $p \mathrm{CA}$ responds to dilute acid pretreatment. To achieve this, we screened a diverse set of corn stover samples to a range of pretreatment severities. The six corn stover genotypes (Fig. 1) were selected based on their diversity in $p$ CA content, which ranged from 63.8-107.2 $\mathrm{mg} p \mathrm{CA} / \mathrm{g}$ lignin and Klason lignin content (12.9-19.3\% by mass). The selected genotypes fall into two general categories of high-lignin, low- $p$ CA (B14A, NK807, 5220) and low-lignin, high- $p$ CA (B7, NC368, F431). Critically, as shown in Fig. 1, $p$ CA can comprise more than $10 \%$ by mass of the aromatic content of lignins in graminaceous monocots such as maize. For an integrated biorefinery processing corn stover, this represents an enormous reservoir of potentially recoverable and utilizable aromatics. For example, in an integrated biorefinery processing 2000 tonnes/day of corn stover, the range of $p$ CA contents in Fig. 1 represents between 23 and 34 tonnes/day of $p$ CA passing through the biorefinery. 
The six maize genotypes were subjected to dilute acid pretreatment over a range of severities according to the conditions in Table 1. Additionally, the values for the combined severity factor (CSF) are presented using the equation for CSF (based on initial $\mathrm{pH}$ ) and parameters developed by Chum et al. (1990). Conditions for pretreatment were selected based on the CSF range identified by Schell et al. (2003) as achieving glucose hydrolysis yields $>70 \%$ for dilute acid-pretreated corn stover. While the CSF values were within a comparable range, the pretreatment times were much longer (up to $6 \mathrm{hr}$ rather than $<10 \mathrm{~min}$ ) and the initial $\mathrm{pH}$ values were much higher (i.e., lower acid loadings were utilized). The reasons these milder range of conditions were chosen are so that (1) these milder acid loadings would allow the reaction to take place in reactors without use of expensive, corrosion-resistance alloys, substantially lowering capital costs, (2) improve the selectivity of xylan hydrolysis to xylose dehydration to furfural, and (3) potentially decrease the hydrolysis of esterified $p \mathrm{CA}$ while simultaneously achieving pretreatment performance targets. It should be noted that not all biomass-pretreatment combinations were employed in this study.

\subsection{Impact of Pretreatment on Cell Wall Composition and Enzymatic Hydrolysis Yields}

The impact of pretreatment conditions on the solubilization of key cell wall components was assessed with a focus on xylan and $\underline{\mathrm{CA}}$. Xylans are a major structural component of the plant cell walls of angiosperms and are involved in non-covalent cross-links between cellulose microfibrils (Ong et al., 2014). Hemicelluloses inhibit the ability of cellulase enzymes to access cellulose within the cell wall structure and reduce the effectiveness of enzyme hydrolysis and, consequently, the overall potential biofuel yield. Hemicellulose removal is typically a target of the biomass deconstruction process either during pretreatment by hydrolysis and/or solubilization or during enzymatic hydrolysis by xylanolytic enzymes. During dilute acid pretreatments, acid catalyzes the hydrolysis of the glycosidic bonds with xylans resulting in their removal from the cell wall in the form of its constituent oligomers and monomers. Dilute acid pretreatment also results in the melting and redistribution of lignin within the cell wall (Donohoe et al., 2008; Selig et al., 2007). These two outcomes of dilute acid pretreatment allow the cellulase enzymes better access to cellulose within the biomass, resulting in higher total glucose hydrolysis yields. The amount of xylan removed and solubilized during dilute acid pretreatments is commonly used to evaluate the effectiveness of different dilute acid pretreatment conditions and how we expect 
these conditions to influence the maximum obtainable hydrolysis yield from pretreated corn stover (Schell et al., 2003; Yang \& Wyman, 2004).

The primary contributors to the effectiveness of a dilute acid pretreatment are temperature, time, and acid catalyst concentration. As all the experiments were performed at $180^{\circ} \mathrm{C}$, the time and initial $\mathrm{pH}$ are the only two factors that will be contributing to different responses in this work. The results for changes to xylan content are presented in Fig. 2 and show several notable trends. Specifically, when all samples are pooled, the xylan solubilization can be shown to be a strong, significant function of time, with longer pretreatment times resulting in significantly higher levels of xylan solubilization (Fig. 2B). For the 3-hr pretreatment times, where the most data points were collected, even the minor differences in the initial $\mathrm{pH}$ have a significant $(p<$ 0.001) impact on the xylan solubilization during pretreatment (Fig. 2C).

In prior work, $p \mathrm{CA}$ solubilization during acidic treatments has been studied primarily to understand its role as an inhibitor of cellulases during enzymatic hydrolysis when present in hydrolysate liquors (Martin \& Akin, 1988; Theodorou et al., 1987), or when intact in the cell wall (Hartley, 1972; Hartley \& Jones, 1978). The effects of pretreatment-solubilized $p$ CA on microbial fermentation have also been studied (Chundawat et al., 2010; Du et al., 2010; Mitchell et al., 2014). Finally, $p$ CA solubilization has also been used as a standard analytical tool for the analysis of $p$ CA-polysaccharide esters (Hatfield et al., 2008). However, a detailed information on the kinetics or hydrolysis of $p \mathrm{CA}$-lignin esters during chemical pretreatments is scarce in the literature. As one example, it is known that hydrolysis using $0.1 \mathrm{M}$ trifluoroacetic acid at $100^{\circ} \mathrm{C}$ for 1 hour is capable of hydrolyzing glycosidic bonds within arabinoxylan while preserving the ester bond of $p$ CA acylated to arabinosyl subunits (Hatfield et al., 2008). One prior study showed that $p \mathrm{CA}$ was the most abundant aromatic monomer in liquors derived from dilute acid pretreatment of corn stover with the quantified content equivalent to $16.7 \mathrm{mg} p \mathrm{CA} / \mathrm{g}$ original Klason lignin (Chundawat et al., 2010). The pretreatment conditions in this study were $190^{\circ} \mathrm{C}$, an estimated initial $\mathrm{pH}$ of 0.7 , and a residence time of $<10$ minutes. Other work in sugarcane bagasse has shown that pretreatment under acidic conditions resulted in less $p C A$ in the hydrolysate when compared to steam pretreatment, and pretreatment in the presence of $\mathrm{SO}_{2}$ due to the further degradation of $p \mathrm{CA}$ and other aromatic acids during pretreatment (Martin et al., 2002). 
In the present work, dilute acid pretreatment is shown to result in extensive hydrolysis of $p \mathrm{CA}$ (Fig. 3). The results for $p \mathrm{CA}$ removal correspond with those for xylan removal and show that both time and initial $\mathrm{pH}$ are key parameters in its removal from the cell wall. Under the most severe pretreatment conditions (6-hr pretreatment time), a 6.5-fold reduction in $p$ CA content was observed. As with the xylan solubilization, pretreatment time was found to correlate to reduction in $p$ CA content (Fig. 3B). For every genotype subjected to 3-hr pretreatments, the initial $\mathrm{pH}$ had a statistically significant impact on the $p$ CA removal (Fig. 3C).

Enzymatic hydrolysis of cellulose to glucose provides an assessment of the relative recalcitrance of the cell wall and, additionally, is a key step determining the economic feasibility of ethanol production from lignocellulosic biomass. Enzymatic hydrolysis is considered to be one of the largest contributions to the operating cost in a biorefinery with enzymes estimated to contribute up to $\$ 1$ per gallon of ethanol produced (Kumar \& Murthy, 2013). It can be observed that, with the exception of the B7, the low-lignin biomass (F431, NC368) generally exhibited higher hydrolysis yields than the high-lignin biomass (B14A, NK807, 5220). As lignin is not removed during batch dilute acid pretreatments, but only melted and redistributed, this could be expected as redeposited lignin can prevent hydrolytic enzymes from accessing glycosidic bonds within cellulose (Selig et al., 2007). Comparable to the impacts of dilute acid pretreatment on xylan and $p \mathrm{CA}$ removal, longer dilute acid pretreatment times result in greater hydrolysis yields due to increases hemicellulose removal during pretreatment. However, unlike the other results following three-hour pretreatment whereby differences in the initial $\mathrm{pH}$ resulted in differences in xylan (Fig. 2B) and $p$ CA solubilization (Fig. 3B), a significant difference in hydrolysis yields between differing $\mathrm{pH}$ pretreatments was not observed (Fig. 4C). This suggests that higher severity pretreatments at lower $\mathrm{pH}$ results in addition, unquantified changes to the biomass beyond xylose and $p \mathrm{CA}$ removal which affect hydrolysis yields.

\subsection{Correlation of Data}

Select trends within the data set are plotted in order to highlight key relationships between pretreatment outcomes (Fig. 5). The first key observation is that xylan solubilization is generally correlated to glucose hydrolysis yields across all pretreatment conditions and genotypes (Fig. 5A). Xylan removal (or alternatively xylose generation) has been used as a proxy for the extent of pretreatment and has been shown to exhibit correlations to the enzymatic hydrolysis yields 
during dilute acid pretreatments (Yao et al., 2018), with one study showing strong linear correlations between xylan removal and enzymatic hydrolysis yields in corn stover subjected to dilute acid pretreatment in both batch and flowthrough reactor configurations (Yang \& Wyman, 2004). Other work in a continuous pilot-scale reactor showed that rather than exhibiting a continuous linear increase in hydrolysis yields with xylan removal that the glucose hydrolysis yields in dilute acid-pretreated corn stover reached a maximum at xylan solubilization levels of $>60 \%$ (Schell et al., 2003). The data show that for each of the six genotypes, xylan solubilization above 50\% did not result substantial improvements in glucose hydrolysis yields (Fig. 5A) as has been shown in previous work (Schell et al., 2003; Jeoh et al., 2007). As shown previously, the high-lignin biomass generally exhibited lower yields than the low-lignin biomass.

The glucose hydrolysis yields are plotted against the $p \mathrm{CA}$ content for all biomasspretreatment combinations to highlight the balance between achieving high hydrolysis yields versus hydrolyzing $p \mathrm{CA}$ during the pretreatment (Fig. 5B). These results show a sigmoidal relationship between these two variables and it can be observed that at least two biomasspretreatment combinations (highlighted in Fig. 5B with a " $\star$ ") are capable of achieving hydrolysis yields $>90 \%$ while retaining more than $50 \mathrm{mg} p \mathrm{CA} / \mathrm{g}$ original Klason lignin. It is notable that both genotypes (NC368 and F431) are from the low-lignin, high- $p$ CA category suggesting that the two objectives of high sugar yields and high $p C A$ retention can be achieved simultaneously by selection of appropriate feedstock-pretreatment combinations. The conditions used for pretreatment $\left(3 \mathrm{hr}, \mathrm{pH}_{0}=3.36,180^{\circ} \mathrm{C}\right)$ at the higher initial $\mathrm{pH}$ was shown to result in less $p$ CA removal (Fig. 3C) while not having a significant impact on hydrolysis yields (Fig. 4C).

The next within-dataset correlation exhibiting notable trends is the relationship between $p$ CA solubilization and xylan solubilization (Fig. 5C). For this, a strong linear correlation can be observed for all biomass samples except for samples with $p$ CA solubilization greater than $\sim 65 \%$. This indicates that under the conditions tested the rate of hydrolysis of an acid-catalyzed $p$ CAlignin ester linkage is of the same order of magnitude as the hydrolysis of a glycosidic bond within xylan. In the next section, these kinetics for $p C A$ ester hydrolysis are explored in more detail. 


\subsection{Kinetics of $p C A$ Ester Hydrolysis}

In order to further understand the kinetics of $p \mathrm{CA}$ ester hydrolysis, a kinetic study was next performed using trans- $p \mathrm{CA}$ methyl ester as a model compound with the results compared to those from corn stover. The hydrolysis of phenolic esters has been studied since at least 1937 when Hammett developed a relationship relating the rate of reaction of substituted aromatic compounds to the structure of their unique substitution (Hammett, 1937). Kinetic models based on modified versions of the Hammett equation have been developed to explain the hydrolysis of various esters catalyzed by both dilute and concentrated acid (Taft, 1952; Yates, 1971). In addition to these models these kinetic principles have been used to explore the mechanism behind acid-catalyzed ester hydrolysis. Shi et. al. suggest that an ester bond is hydrolyzed by acid according to a two-step mechanism; an alkyl-oxygen protonation which creates an acylium ion followed by a trimolecular hydrolysis (Shi et al., 2015).

In the present work, the $p \mathrm{CA}$ methyl ester was hydrolyzed under conditions comparable to those used for the dilute acid pretreatments of corn stover with times ranging from 2 to 17 hours, initial $\mathrm{pH}$ values of 3.36 and 2.86 , and a temperature of $180^{\circ} \mathrm{C}$. The goal of this set of experiments was to gain insight into the kinetics of the acid-catalyzed hydrolysis of the $p$ CAlignin ester bond responsible for the removal of $p \mathrm{CA}$ during dilute acid pretreatment. Results are plotted as a dimensionless conversion such that all biomass samples with varying $p \mathrm{CA}$ contents could be plotted for comparison (Fig. 6A). Exponential functions were fit to the curves with the assumption that ester hydrolysis kinetics could be approximated by a first-order reaction with respect to $p C A$ concentration with rate constants estimated to be $0.081 \mathrm{hr}^{-1}\left(\mathrm{pH}_{0}=\right.$ $3.36)$ and $0.183 \mathrm{hr}^{-1}\left(\mathrm{pH}_{0}=2.86\right)$. This is consistent with increasing acid catalyst concentration increasing the rate constant as a contributor to activation energy or, alternatively, as a concentration dependence in an elementary rate law. These results can be compared to the $p \mathrm{CA}$ hydrolysis kinetics results pooled from all the biomass-pretreatment conditions screened in this work with $\mathrm{pH}_{0}$ ranging from 2.86 to 3.66 (Fig. 6A). Assuming first-order kinetics for this reaction, the estimated rate constant for xylan hydrolysis and removal from the corn stover is $0.29 \mathrm{hr}^{-1}$. Although this is the same order of magnitude as the rate constant for model compound ester hydrolysis, it is still significantly higher. Potential reasons for this discrepancy could be either differing mechanisms for the hydrolysis of a $p \mathrm{CA}$-lignin ester versus a $p \mathrm{CA}$-methyl ester. A second possibility is that the presence of biomass during the reaction results in generation of 
additional acids during pretreatment through autohydrolysis of acetyl groups within the hemicellulose and further decreases the $\mathrm{pH}$ during the pretreatment. However, it has been shown that $\mathrm{pH}$ decreases in liquid hot water and dilute acid pretreatments do not generally proceed below pH 3.0 (Kapu et al., 2016).

The results of the $p \mathrm{CA}$ ester hydrolysis kinetics can be compared to those of the xylan in corn stover (Fig. 6B). For this, data were selected from all samples and conditions that used a $\mathrm{pH}_{0} \geq 3.16$ as the lower $\mathrm{pH}$ condition resulted in substantially higher xylan hydrolysis (Fig. 2C). An exponential curve is fit to the data under the assumption that xylan solubilization can be approximated by first-order reaction kinetics yielding a rate constant of $0.187 \mathrm{hr}^{-1}$ which is of the same order of magnitude as the $p \mathrm{CA}$ hydrolysis (Fig. 6A). This finding of comparable rate constants for $p \mathrm{CA}$ and xylan hydrolysis is supported by the previous observation of a linear correlation between xylan and $p \mathrm{CA}$ removal during pretreatment (Fig. 5C). Xylan removal from the cell wall involves hydrolysis of cellulose-associated insoluble xylan to yield water-soluble oligomeric xylan. Unless this xylan is further hydrolyzed to monomeric xylose, these xylooligomers may be able to adsorb back onto the cellulose. More complex models for xylan hydrolysis within biomass have been developed that include two pools of xylan ("slow-reacting" and "fast-reacting") that first hydrolyze to oligomers followed by hydrolysis of oligomers to monomers with each of these reactions exhibiting differing rate constants that are elementary with respect to xylan and acid concentration (Schell et al., 2003). Using the expressions developed by Schell et al. (Schell et al., 2003) for the hydrolysis of xylan during dilute acid pretreatment of corn stover, values for the rate constants for "slow-reacting" xylan (presumably the rate-limiting step) are calculated to be $0.48 \mathrm{hr}^{-1}$ for a $\mathrm{pH}$ of 2.86 and $0.152 \mathrm{hr}^{-1}$ for a $\mathrm{pH} 3.36$, which bounds the estimated rate constant of $0.127 \mathrm{hr}^{-1}$.

\section{Conclusions}

A panel of six diverse maize genotypes exhibiting a range of $p \mathrm{CA}$ and lignin contents was used to study the relationship between dilute acid pretreatment conditions and the biomass response to pretreatment with the goals understanding $p \mathrm{CA}$ ester hydrolysis kinetics during pretreatment. For this we employed a range of pretreatment conditions utilizing mild dilute acid (initial $\mathrm{pH} \geq 2.86$ ) over times ranging from 45 minutes to 6 hours at $180^{\circ} \mathrm{C}$. Strong correlations between xylan solubilization and $p C A$ solubilization were identified. Using a model $p C A$-ester, 
kinetic parameters for acid-catalyzed ester hydrolysis were estimated and were of the same order of magnitude as those for xylan hydrolysis under comparable conditions.

E-supplementary data of this work can be found in the online version of the paper.

\section{Acknowledgements}

This work was supported by AFRI competitive grant no. 2018-67009-27900/1015055 from the USDA National Institute of Food and Agriculture.

\section{References}

1. Carlson, D.C. 2020. System for the treatment of biomass. U.S. Pat. No. 10,533,203 B2.

2. Carpita, N.C. 1996. Structure and biogenesis of the cell walls of grasses. Annu. Rev.Plant Physiol. Plant Mol. Biol., 47, 445-476.

3. Chum, H.L., Johnson, D.K., Black, S.K. 1990. Organosolv pretreatment for enzymic hydrolysis of poplars. 2. Catalyst effects and the combined severity parameter. Ind. Eng. Chem. Res., 29(2), 156-162.

4. Chundawat, S.P.S., Vismeh, R., Sharma, L.N., Humpula, J.F., da Costa Sousa, L., Chambliss, C.K., Jones, A.D., Balan, V., Dale, B.E. 2010. Multifaceted characterization of cell wall decomposition products formed during ammonia fiber expansion (AFEX) and dilute acid based pretreatments. Biores. Technol., 101(21), 8429-8438.

5. Donohoe, B.S., Decker, S.R., Tucker, M.P., Himmel, M.E., Vinzant, T.B. 2008. Visualizing lignin coalescence and migration through maize cell walls following thermochemical pretreatment. Biotechnol. Bioeng., 101(5), 913-25.

6. Du, B., Sharma, L.N., Becker, C., Chen, S.F., Mowery, R.A., van Walsum, G.P., Chambliss, C.K. 2010. Effect of varying feedstock-pretreatment chemistry combinations on the formation and accumulation of potentially inhibitory degradation products in biomass hydrolysates. Biotechnol. Bioeng., 107(3), 430-40.

7. Hammett, L.P. 1937. The effect of structure upon the reactions of organic compounds. Benzene derivatives. J. Am. Chem. Soc., 59(1), 96-103.

8. Hartley, R.D. 1972. p-Coumaric and ferulic acid components of cell walls of ryegrass and their relationships with lignin and digestibility. J. Sci. Food Agric., 23(11), 1347-1354. 
9. Hartley, R.D., Jones, E.C. 1978. Phenolic components and degradability of the cell walls of the brown midrib mutant, bm3, of Zea mays. J. Sci. Food Agric., 29(9), 777-782.

10. Hatfield, R.D., Marita, J.M., Frost, K. 2008. Characterization of $p$-coumarate accumulation, p-coumaroyl transferase, and cell wall changes during the development of corn stems. J. Sci. Food Agric., 88(14), 2529-2537.

11. Hatfield, R.D., Ralph, J., Grabber, J.H. 1999. Cell wall cross-linking by ferulates and diferulates in grasses. J. Sci. Food Agric., 79(3), 403-407.

12. Jeoh, T., Ishizawa, C.I., Davis, M.F., Himmel, M.E., Adney, W.S., Johnson, D.K. 2007. Cellulase digestibility of pretreated biomass is limited by cellulose accessibility. Biotechnol. Bioeng., 98(1), 112-122.

13. Kalami, S., Arefmanesh, M., Master, E., Nejad, M. 2017. Replacing 100\% of phenol in phenolic adhesive formulations with lignin. J. Appl. Polym. Sci., 134(30), 45124.

14. Kalami, S., Chen, N., Borazjani, H., Nejad, M. 2018. Comparative analysis of different lignins as phenol replacement in phenolic adhesive formulations. Ind. Crops Prod., 125, 520528.

15. Kapu, N.S., Yuan, Z., Chang, X.F., Beatson, R., Martinez, D.M., Trajano, H.L. 2016. Insight into the evolution of the proton concentration during autohydrolysis and dilute-acid hydrolysis of hemicellulose. Biotechnol. Biofuels, 9, 224.

16. Kumar, D., Murthy, G.S. 2013. Stochastic molecular model of enzymatic hydrolysis of cellulose for ethanol production. Biotechnol. Biofuels, 6(1), 63.

17. Li, M., Heckwolf, M., Crowe, J.D., Williams, D.L., Magee, T.D., Kaeppler, S.M., de Leon, N., Hodge, D.B. 2015. Cell-wall properties contributing to improved deconstruction by alkaline pre-treatment and enzymatic hydrolysis in diverse maize (Zea mays L.) lines. J. Exp. Bot., 66(14), 4305-4315.

18. Li, M., Williams, D.L., Heckwolf, M., de Leon, N., Kaeppler, S., Sykes, R.W., Hodge, D. 2017. Prediction of cell wall properties and response to deconstruction using alkaline pretreatment in diverse maize genotypes using Py-MBMS and NIR. Bioenerg. Res., 10(2), 329-343.

19. Mansouri, N.-E., Salvadó, J. 2006. Structural characterization of technical lignins for the production of adhesives: Application to lignosulfonate, kraft, soda-anthraquinone, organosolv and ethanol process lignins. Ind. Crops Prod., 24, 8-16. 
20. Martin, C., Galbe, M., Nilvebrant, N.O., Jonsson, L.J. 2002. Comparison of the fermentability of enzymatic hydrolyzates of sugarcane bagasse pretreated by steam explosion using different impregnating agents. Appl. Biochem. Biotechnol., 98-100, 699-716.

21. Martin, S.A., Akin, D.E. 1988. Effect of phenolic monomers on the growth and betaglucosidase activity of Bacteroides ruminicola and on the carboxymethylcellulase, betaglucosidase, and xylanase activities of Bacteroides succinogenes. Appl. Environ. Microbiol., 54(12), 3019-22.

22. Mitchell, V.D., Taylor, C.M., Bauer, S. 2014. Comprehensive analysis of monomeric phenolics in dilute acid plant hydrolysates. Bioenerg. Res., 7(2), 654-669.

23. Ong, R.G., Chundawat, S.P.S., Hodge, D.B., Keskar, S., Dale, B.E. 2014. Linking plant biology and pretreatment: Understanding the structure and organization of the plant cell wall and interactions with cellulosic biofuel production, in: Plants and BioEnergy, (Ed.) C. McCann, M.S. Buckeridge, N.C. Carpita, Springer, pp. 231-253.

24. Ragauskas, A.J., Beckham, G.T., Biddy, M.J., Chandra, R., Chen, F., Davis, M.F., Davison, B.H., Dixon, R.A., Gilna, P., Keller, M., Langan, P., Naskar, A.K., Saddler, J.N., Tschaplinski, T.J., Tuskan, G.A., Wyman, C.E. 2014. Lignin valorization: Improving lignin processing in the biorefinery. Science, 344(6185), 1246843.

25. Schell, D.J., Farmer, J., Newman, M., McMillan, J.D. 2003. Dilute-sulfuric acid pretreatment of corn stover in pilot-scale reactor. Appl. Biochem. Biotechnol., 105(1), 69-85.

26. Selig, M.J., Viamajala, S., Decker, S.R., Tucker, M.P., Himmel, M.E., Vinzant, T.B. 2007. Deposition of lignin droplets produced during dilute acid pretreatment of maize stems retards enzymatic hydrolysis of cellulose. Biotechnol. Prog., 23(6), 1333-9.

27. Shanks, B. H. and Keeling, P. L. 2017. Bioprivileged molecules: Creating value from biomass. Green Chem, 19(14), 3167-3430.

28. Shi, H., Wang, Y., Hua, R. 2015. Acid-catalyzed carboxylic acid esterification and ester hydrolysis mechanism: Acylium ion as a sharing active intermediate via a spontaneous trimolecular reaction based on density functional theory calculation and supported by electrospray ionization-mass spectrometry. Phys. Chem. Chem. Phys., 17(45), 30279-30291.

29. Sluiter, A., Hames, B., Ruiz, R., Scarlata, C., Sluiter, J., Templeton, D. 2008. Determination of ash in biomass. NREL/TP-510-42622 NREL Laboratory Analytical Procedure. National Renewable Energy Laboratory, Golden, CO, USA. 
30. Song, Y., Wang, Z., Yan, N., Zhang, R., Li, J. 2016. Demethylation of wheat straw alkali lignin for application in phenol formaldehyde adhesives. Polymers, 8(6), 209.

31. Taft, R.W. 1952. Linear free energy relationships from rates of esterification and hydrolysis of aliphatic and ortho-substituted benzoate esters. J. Am. Chem. Soc., (11), 2729-2732.

32. Theodorou, M.K., Gascoyne, D.J., Akin, D.E., Hartley, R.D. 1987. Effect of phenolic acids and phenolics from plant cell walls on rumenlike fermentation in consecutive batch culture. Appl. Environ. Microbiol., 53(5), 1046-1050.

33. Warner, E., Schwab, A., Bacovsky, D. 2017. 2016 Survey of Non-Starch Alcohol and Renewable Hydrocarbon Biofuels Producers. NREL/TP-6A10-65519. National Renewable Energy Laboratory, Golden, CO, USA.

34. Yang, B., Wyman, C.E. 2004. Effect of xylan and lignin removal by batch and flowthrough pretreatment on the enzymatic digestibility of corn stover cellulose. Biotechnol. Bioeng., 86(1), 88-95.

35. Yao, L., Yang, H., Yoo, C.G., Pu, Y., Meng, X., Muchero, W., Tuskan, G.A., Tschaplinski, T., Ragauskas, A.J. 2018. Understanding the influences of different pretreatments on recalcitrance of Populus natural variants. Biores. Technol., 265, 75-81.

36. Yates, K. 1971. Kinetics of ester hydrolysis in concentrated acid. Acc. Chem. Res., 4(4), 136144. 


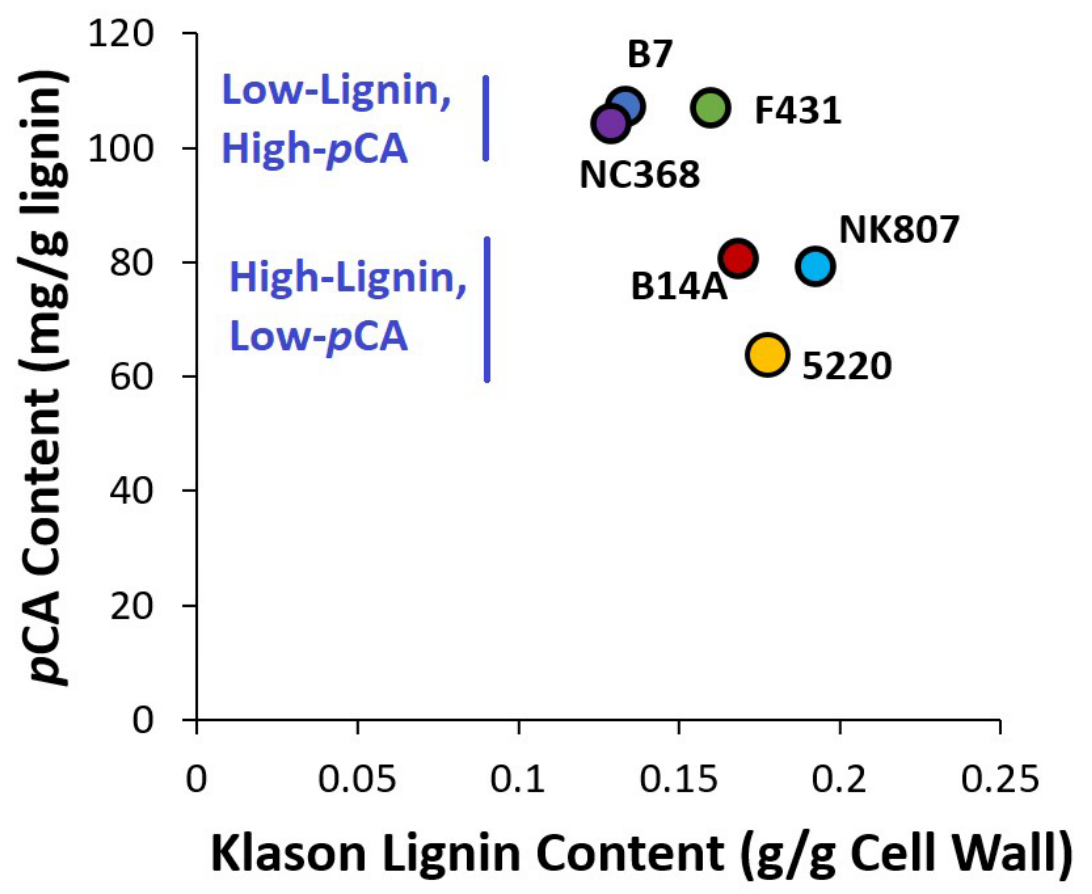



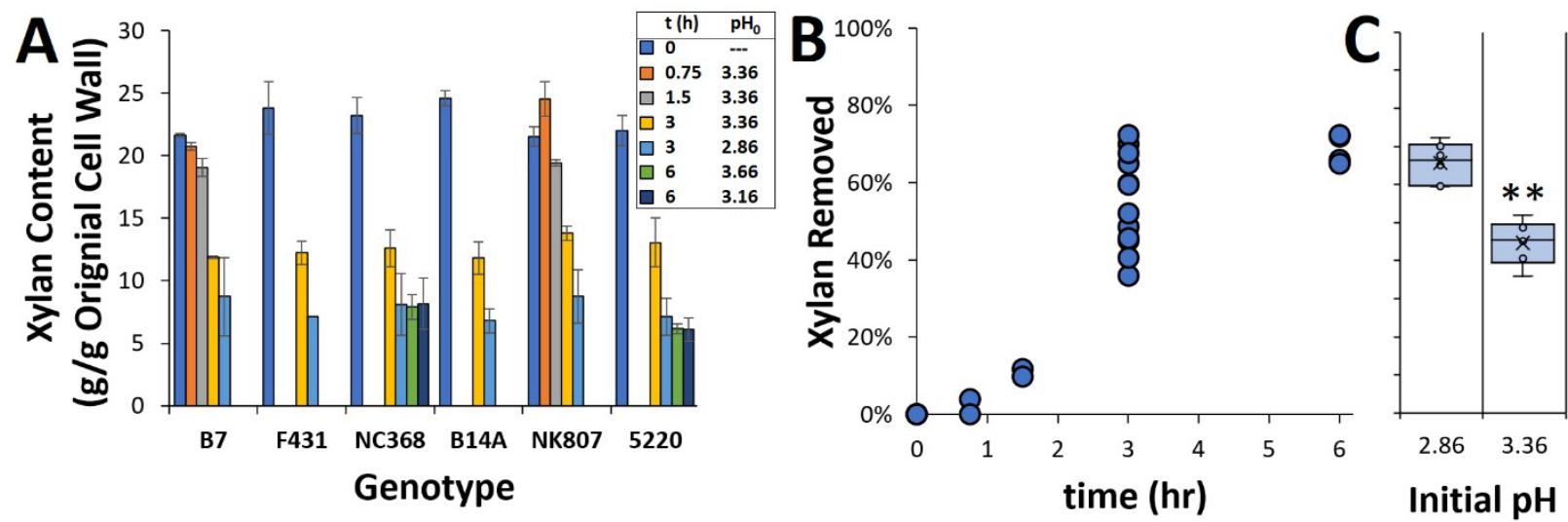

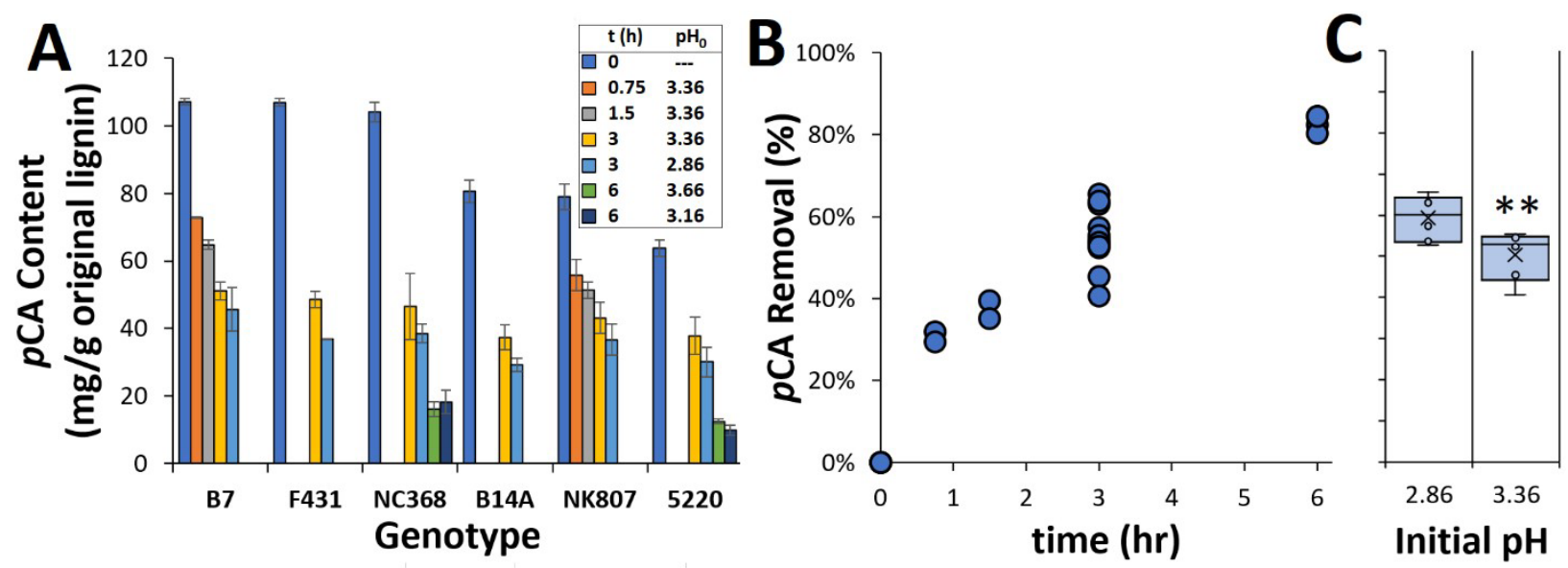

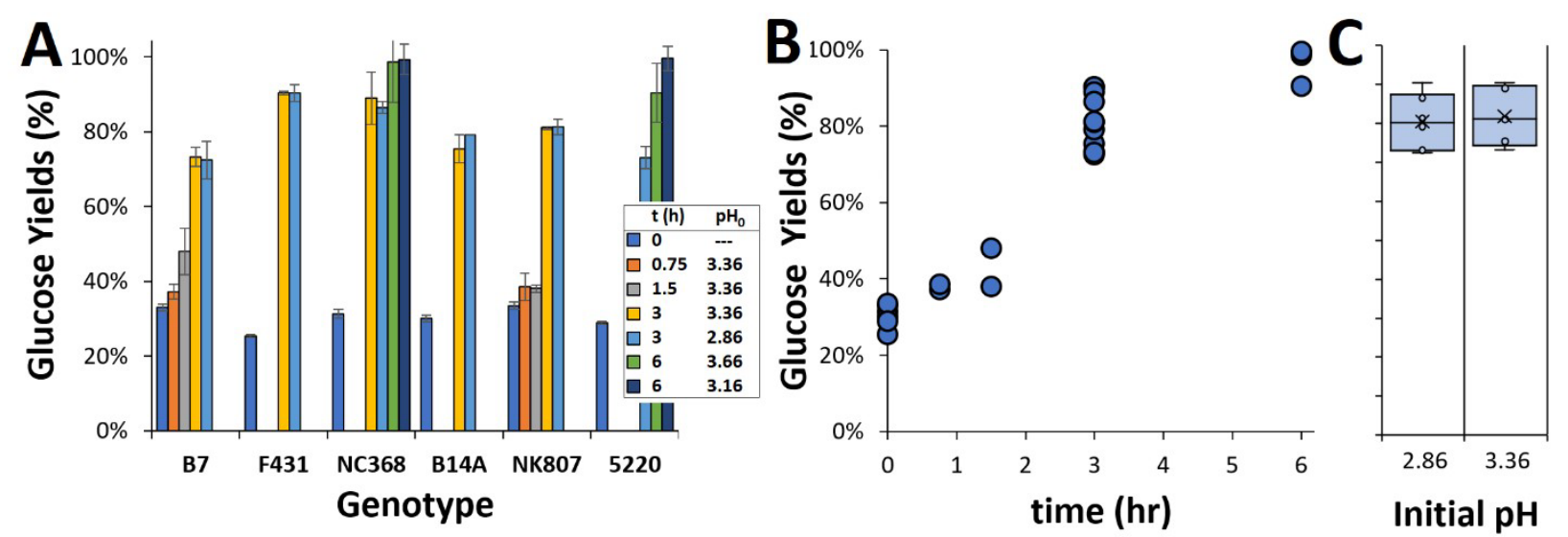

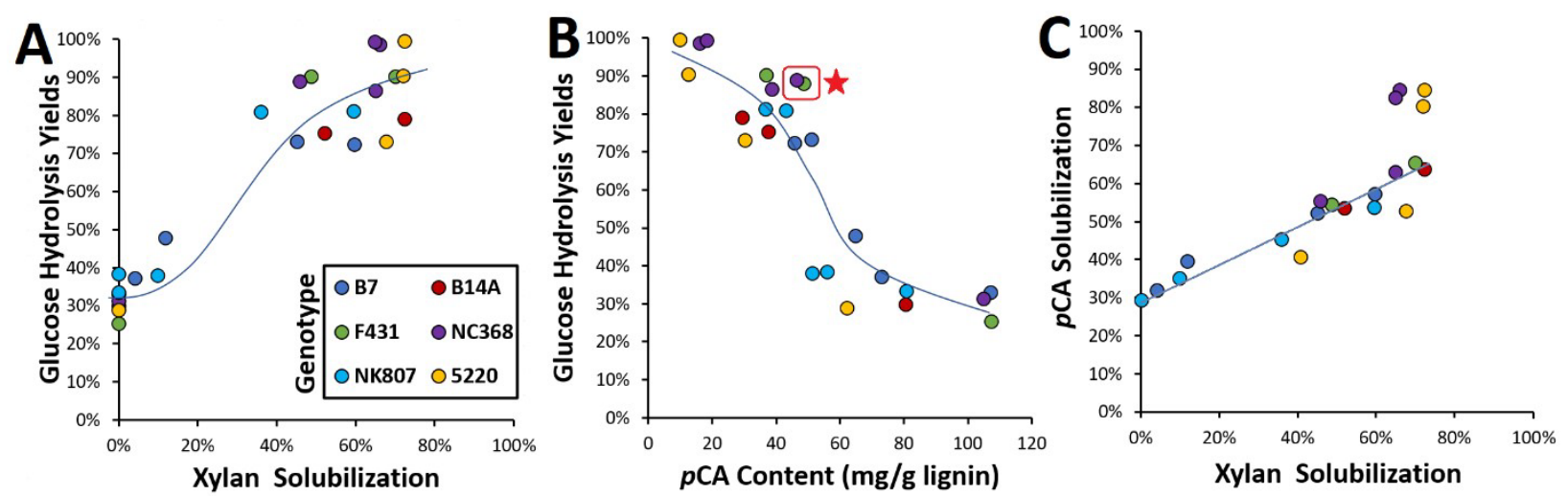

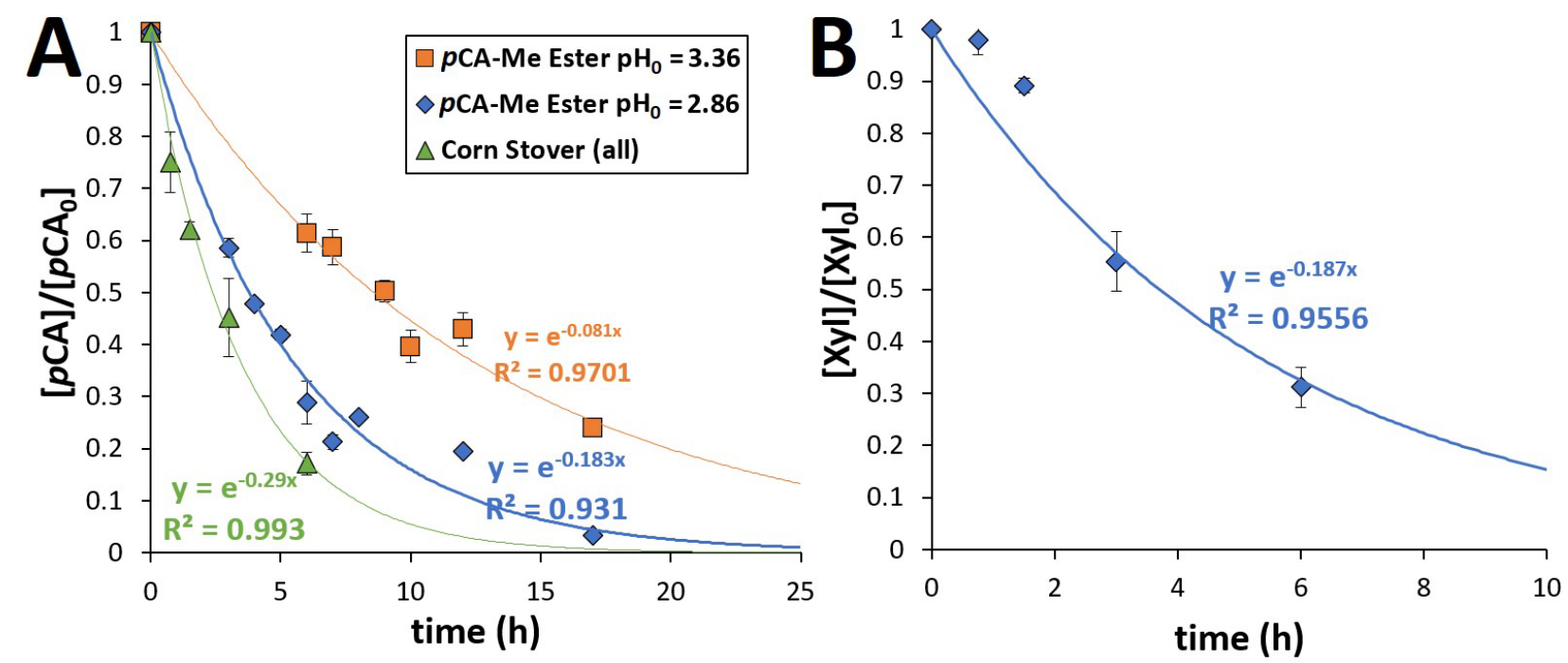


\begin{tabular}{cccc}
\hline Time (hr) & Initial $\mathbf{p H}\left(\mathbf{p H}_{\mathbf{0}}\right)$ & Temperature & $\mathbf{C S F}$ \\
\hline 0.75 & 3.36 & $180^{\circ} \mathrm{C}$ & 0.65 \\
1.5 & 3.36 & $180^{\circ} \mathrm{C}$ & 0.95 \\
3 & 3.36 & $180^{\circ} \mathrm{C}$ & 1.25 \\
3 & 2.86 & $180^{\circ} \mathrm{C}$ & 1.75 \\
6 & 3.66 & $180^{\circ} \mathrm{C}$ & 1.25 \\
6 & 3.16 & $180^{\circ} \mathrm{C}$ & 1.75 \\
\hline
\end{tabular}

Table 1: Dilute acid pretreatment conditions and the calculated value for the combined severity factor (CSF). 